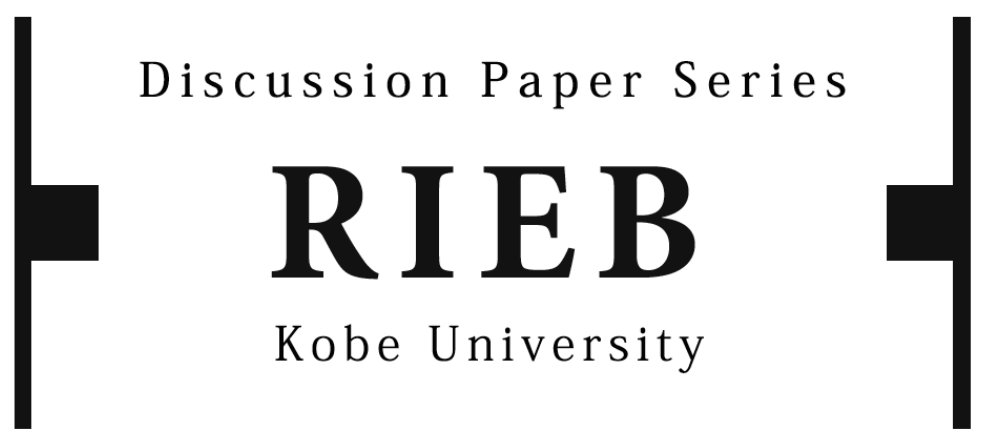

DP2017-23

Examining Japanese Women's Preferences for a New Style of Postnatal Care Facility and Its Attributes

\author{
Junyi SHEN \\ Takako NAKASHIMA \\ Izumi KARASAWA \\ Tatsuro FURUI \\ Kenichiro MORISHIGE \\ Tatsuyoshi SAIJO
}

September 28, 2017

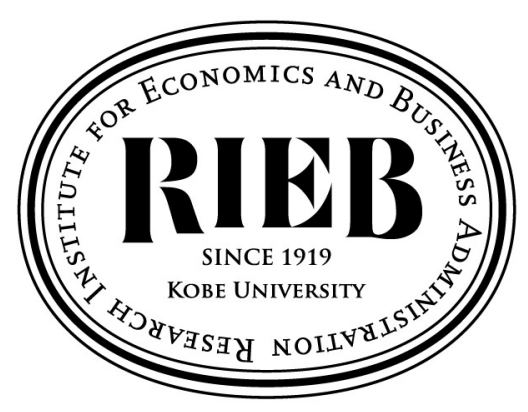

Research Institute for Economics and Business Administration Kobe University 


\title{
Examining Japanese women's preferences for a new style of postnatal care facility and its attributes
}

\author{
Junyi Shen $^{\mathrm{a}, \mathrm{b}, *}$, Takako Nakashima ${ }^{\mathrm{c}}$, Izumi Karasawa ${ }^{\mathrm{d}}$, \\ Tatsuro Furui $^{\mathrm{e}}$, Kenichiro Morishige ${ }^{\mathrm{e}}$, and Tatsuyoshi Saijo ${ }^{\mathrm{f}, \mathrm{g}}$ \\ ${ }^{a}$ Research Institute for Economics and Business Administration, Kobe University \\ b School of Economics, Shanghai University

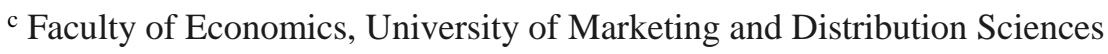 \\ ${ }^{d}$ Department of Midwifery, Gifu University of Medical Science \\ e Department of Obstetrics and Gynecology, Graduate School of Medicine, Gifu University \\ ${ }^{\mathrm{f}}$ Research Institute for Humanity and Nature, Kyoto \\ ${ }^{\mathrm{g}}$ Research Institute for Future Design, Kochi University of Technology \\ * Corresponding author. Research Institute for Economics and Business Administration, Kobe \\ University, 2-1 Rokkodai, Kobe, Hyogo 6578501, Japan. E-mail: shen@rieb.kobe-u.ac.jp.
}

\begin{abstract}
Perinatal care in rural Japan is currently facing a crisis because of the lack of medical staff, especially obstetricians. In this study, a new style of postnatal care facility that combines both medical and nonmedical support is considered. Contrary to most postnatal care facilities in Japan, this new postnatal care facility accepts a puerperant from the cooperating maternity facility soon after birth ( $\leq 2$ days). We conducted a hypothetical-choice experiment to investigate whether this new postnatal care facility could be accepted by women in Gero City, Hida, Gifu Prefecture and how these women evaluate different kinds of postnatal care services. The results show that after a 2-day hospital stay, women from Gero City preferred to move to the new postnatal care facility over the other alternatives (continued hospitalization or discharge home). In addition, the estimated choice probabilities for selecting the postnatal care facility under different scenarios show a high level of acceptance for this new postnatal care facility.
\end{abstract}

Keywords: postnatal care facility; obstetrics; preference; choice experiment; Japan 


\section{Background}

Perinatal care in rural Japan is currently facing a crisis because of the lack of obstetricians. In most rural areas, the number of facilities that can manage childbirth is decreasing and the number of areas where delivery must be occur at a remote facility is increasing. This trend will likely become increasingly common in the future. After parturition at a remote place, the mother returns to her residence and cares for her child. However, in rural areas, even when exposed to the mental stress of childrearing and the physical stress of puerperium, there is no perinatal facility in the vicinity and only insufficient care can be received. This slows the recovery of puerperal health and causes mother-to-child problems such as maternity blues and abuse. Therefore, postnatal care facilities that can address both the mental and physical stress of the mother and support better parent-child relationships are especially essential in these rural areas.

The postnatal care facility considered in this study combines both medical and nonmedical support, and this is a new style that differs from those currently used in many areas in Japan. Specifically, this new style of postnatal care facility cooperates with the maternity facility to accept the puerperant soon after delivery ( $\leq 2$ days). The facility is generally near the puerperant's place of residence, and the puerperant stays there during puerperium. Because the postnatal care facility cooperates with the maternity facility even after discharge, the puerperant can still receive examinations from the doctors of the maternity facility. Furthermore, in addition to postpartum care, prenatal care (e.g., pregnancy medical examinations) is also administered by the new postnatal care facility. This is partly because a pregnant woman might feel more comfortable if she can receive prenatal and postnatal care at the same facility. In contrast to this new style of postnatal care facility, many other facilities in Japan generally accept puerperants who have been discharged from a maternity facility after a normal hospitalization period (5-6 days).

The current study focuses on the problems of providing obstetrics services in the Hida, Gifu Prefecture, Japan. Hida is located in a mountainous region (see Figure 1), consists of 3 cities (Takayama City, Gero City, and Hida City) and 1 village (Shirakawa Village), and has an area of 4,177.99 square kilometers. There were 149,072 people in Hida area according to the 2015 Population Census of Japan. The population density of Hida area is 35.7 persons $/ \mathrm{km}^{2}$, which is about one-tenth of Japan (341 persons $/ \mathrm{km}^{2}$ ). There are 4 maternity facilities in Hida: 1 hospital (Takayama Red Cross Hospital) and 2 clinics in Takayama City, and 1 hospital (Gifu Prefectural Gero Hospital; herein referred to as Gero Hospital) in Gero City. In 2012, there were 1,174 births in Hida, including 
768 in Takayama City, 220 in Gero City, 170 in Hida City, and 16 in Shirakawa Village.

There are 2 problems with providing obstetrics services in Hida. First, because of the small number of births, it is difficult to widely distribute maternity facilities across this area while maintaining safety. Second, it is generally believed that at least 4 obstetricians and 2 doctors in other departments (e.g., pediatrics and anesthesiology) are essential to maintaining a safe maternity facility and obstetrics service. However, the small number of births in Gero City makes it extremely difficult to arrange a maternity facility with 4 obstetricians. Note that, from a financial standpoint, 400-600 births/year are needed to maintain a maternity facility in Japan with 4 obstetricians. Furthermore, Takayama Red Cross Hospital has been designated as perinatal medical center for mothers and children and accepts pregnant women who need advanced medical care, but Gero Hospital faces a serious shortage of obstetricians. Currently, there is only one 70 -year-old obstetrician who is in charge of childbirth at Gero Hospital; therefore, managing a risky birth is impossible. Accordingly, it is considered that Gero Hospital should cooperate with Takayama Red Cross Hospital and change its status from being a maternity facility to a new postnatal care facility.

The primary purpose of this paper is to investigate whether this new style of postnatal care facility could be accepted by the women of Gero City and how they would evaluate the different kinds of postnatal care services available. We conducted a hypothetical-choice experiment to obtain data on eliciting women's preferences regarding the new postnatal care facility and its attributes. We chose mothers with toddlers at 9 nursery schools in Gero City as the survey target because women who have had children know their needs during puerperium.

The remainder of the paper is organized as follows. The next section provides a detailed literature review. We then describe the methodology, including choice model specification, choice experiment design, data collection, and sample description. After that, the results and discussion are presented. Finally, we draw our conclusions and suggest potential avenues for future research. 


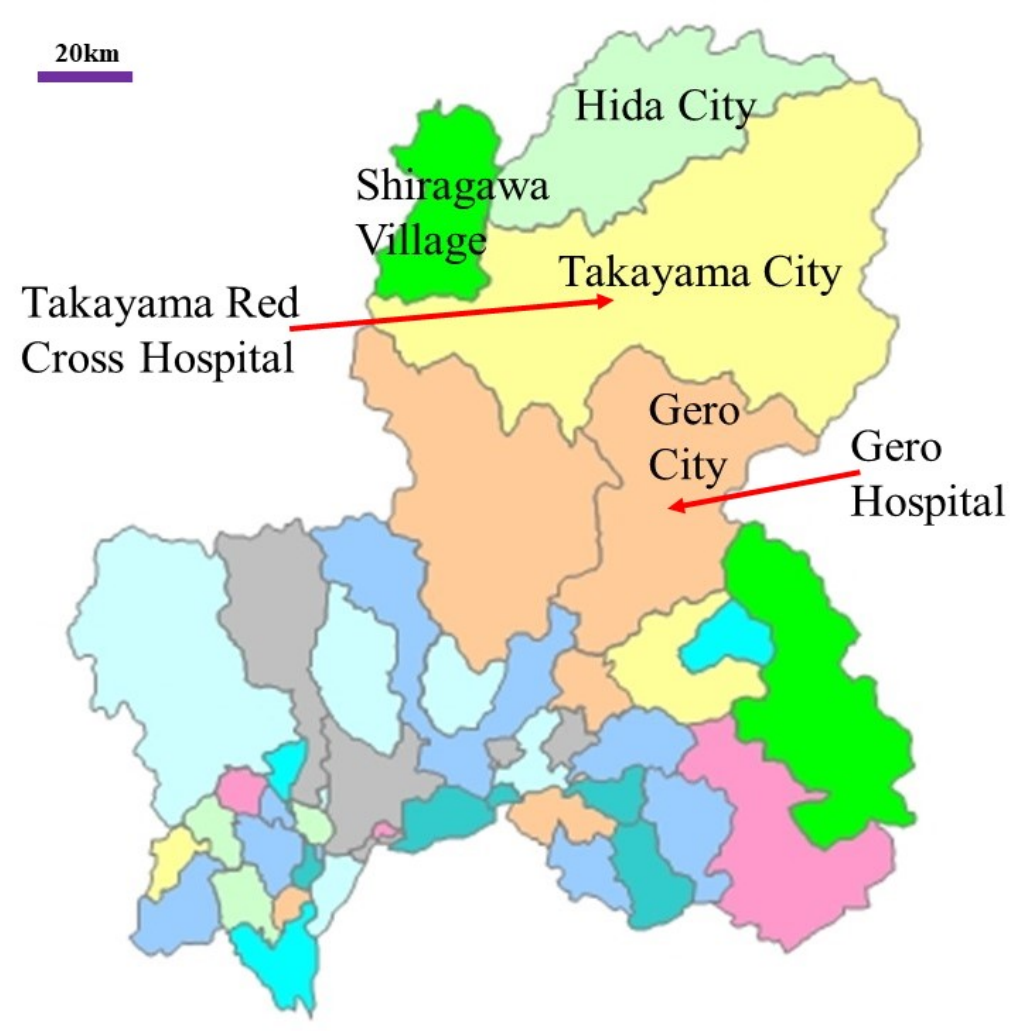

Figure 1. Map of Hida

\section{Literature review}

There are a number of studies on different issues related to postnatal care in the literature. The importance of postnatal care was mentioned by Bastos and McCourt (2010). They argued that it is very common for women to experience a number of postnatal health problems; however, the impact on a woman's well-being needs to be taken seriously by midwives and other healthcare professionals, and these professionals need to offer sufficient, appropriate support and information to women. In addition, Kurth et al. (2010) analyzed interview and participant observation data on 15 mothers in a postnatal ward in Switzerland and stated that, in order to strengthen family health from the earliest stage after delivery, postnatal care should establish the conditions that enable new mothers to balance the care of their infant and their own needs. Bowers and Cheyne (2016) reported that earlier discharge from a hospital resulted in more women and babies having significant care needs at home. Therefore, increasing community-based postnatal care is needed to maintain the quality and safety of postnatal care.

Regarding the acceptance of postnatal care, Ononokpono et al. (2014) used data from the 2008 
Nigeria Demographic and Health Survey to examine community factors associated with receiving postnatal care in Nigeria and the moderating effects of community factors on the association between individual factors and postnatal care. They found significant variations in postnatal care across communities. Specifically, a Nigerian woman's likelihood of receiving postnatal care is a function of where she resides. Living in communities with a high proportion of educated women and a high proportion of women who delivered in a health facility were significantly associated with an increased likelihood of receiving postnatal care.

Tao et al. (2011) surveyed 2,326 women who gave birth in 2 counties of Anhui Province, China from January 2005 to December 2006. Their results showed that only about 4\% of women received $\geq 1$ postnatal visit at home. The authors provided a range of reasons for the low rates of postnatal care provision and utilization and emphasized political support and funding for postnatal care.

Regarding the content of postnatal care services, Smith (2010) found 12 specific dimensions are extremely important and affect women's satisfaction with postnatal care: support from health professionals, a partner, or social support services; care from a general practitioner and health visitor; receiving advice about contraception, feeding the baby, and maternal health; continuity of care; duration of inpatient stay; home visits; and pain after childbirth. In addition, Johansson et al. (2010) interviewed 21 Swedish-speaking parents who had given birth to their first child after a normal pregnancy and delivery. They reported that parents welcome home-based postnatal care and professional support from midwives, especially for advice on breastfeeding. Slade et al. (2010) stated that postnatal depression is a public health problem that requires intervention, and women should recognize the role of health care professionals in treating postnatal depression and accept their support if they find them easy to relate to on personal matters.

In a single large maternity unit in the south of England, Beake et al. (2010) interviewed 20 women who had a vaginal or cesarean birth to evaluate the impact of the postnatal ward environment, staff attitude, quality and level of support for breastfeeding, unmet information needs, and low expectations for hospital-based postnatal care on well-being. Their findings were that although many women had a good experience on the postnatal ward, there was room for improvement in a number of areas, including the environment, routines of the postnatal ward, communication skills of the staff, consistency in providing breastfeeding support, and providing appropriate and relevant information to support women and their partners in preparing for discharge home. Moreover, Rayner et al. (2010) collected postal survey data from 19 private hospitals and interviewed 11 clinical midwives, 
maternity unit managers, and obstetricians in Victoria, Australia. They found that, compared with public hospitals, private hospitals more often provided single-room accommodations and well-baby nurseries, could accommodate a partner's overnight stay, and allowed women to have a longer length of stay following birth.

In Japan, a number of studies discuss the definition of postpartum early discharge. Because the average discharge time after birth is 5-6 days in Japan, Ichikawa and Iseki (2013) and Sakanashi et al. (2011) defined discharge within 3 days after birth as postpartum early discharge, while Kawahara et al. (2012) extended the number of days to 4. With early discharge in mind, Kawahara et al. (2012) administered a questionnaire and face-to-face interview survey. However, most women in late pregnancy want the number of days spent in the hospital to remain the same. As for the care services that puerperants would most like to receive during hospitalization, the needs of primiparas were high for all items, while parous women wanted advice about breastfeeding and maternal and pediatric health. Ichikawa and Iseki (2013) examined the conditions that allow postpartum early discharge by conducting a survey on support received during current postpartum hospitalization (continued hospitalization). They found that (1) determination of any abnormality in the newborn baby and puerperant was strongly requested during postpartum hospitalization, along with rest time and childcare guidance; and (2) the reason that many puerperants did not want to leave the hospital early was concern that they would not receive the abovementioned care. Based on these results, the authors concluded that, as a condition for early hospital discharge, improving the care administered during hospitalization and providing continuous care to mothers and children after discharge need to be considered.

In addition, Fukai et al. (2015) investigated the utilization of childcare support implemented by the local government of the Arakawa District in Tokyo. The surveyed support services were for mothers and children within 3 months after childbirth. Among these services, a parent-child interchange salon was regarded as important for mothers with nuclear families and mothers who do not have childcare supporters or advisers. Among the new services, the favorite was subsidized tickets for service charges, followed by a classroom for mothers after childbirth. Furthermore, women who preferred postnatal daycare facilities tended to be anxious about childcare and the physical condition of their newborn, and many women who preferred accommodation-style postnatal care facilities were late childbearing mothers. 


\section{Methods}

\subsection{Choice experiment model}

The choice experiment (CE) model is based on random utility theory (Louviere et al., 2000; Shen, 2006). The basic assumption of the random utility approach embodied in CE modeling is that decision makers are utility maximizers: i.e., when given a set of alternatives, the decision maker will choose the alternative that maximizes his or her utility. The alternative utility of an individual $(U)$ cannot be observed; however, it could be assumed to consist of a deterministic component $(V)$ and a random error term $(\varepsilon)$. Formally, an individual $q$ 's utility of alternative $i$ can be expressed as

$$
U_{i q}=V_{i q}+\varepsilon_{i q}
$$

Hence, the probability that individual $q$ chooses alternative $i$ from a particular set $J$ consisting of $j$ alternatives can be written as

$$
P_{i q}=P\left(U_{i q}>U_{j q} ; \forall \quad j(\neq i) \in J\right)=P\left(\varepsilon_{j q}<\varepsilon_{i q}+V_{i q}-V_{j q} ; \forall \quad j(\neq i) \in J\right)
$$

To transform the random utility model into a CE model, certain assumptions about the joint distribution of the vectors of the random error terms are required. If the random error terms are assumed to follow a type I extreme value distribution and be independently and identically distributed across alternatives and cases (or observations), the conditional logit (CL) model is obtained (McFadden, 1974). In the CL model, the choice probability in Equation (2) is expressed as

$$
P_{i q}=\exp \left(V_{i q}\right) / \sum_{j=1}^{J} \exp \left(V_{j q}\right)
$$

Then, making the further assumptions that the deterministic component of utility will be linear and additive $\left(V_{i q}=\beta^{\prime} X_{i q}\right.$ ), the probability in Equation (3) can be given as

$$
P_{i q}=\exp \left(\mu \beta^{\prime} X_{i q}\right) / \sum_{j=1}^{J} \exp \left(\mu \beta^{\prime} X_{j q}\right)
$$

where $\mu$ represents the scale parameter that determines the scale of the utilities. This is typically normalized to 1.0 in CL models. $X_{i q}$ is the explanatory variable matrix of $V_{i q}$, and $\beta^{\prime}$ is the parameter vector associated with matrix $X_{i q}$.

An important assumption of the CL model is the independence of the irrelevant alternatives (IIA) property. This property, which follows from the independently and identically distributed assumption, implies that the relative choice probabilities between any 2 alternatives of choice set $J$ 
are not affected by the inclusion or exclusion of other alternatives in that set. The IIA property is a strict assumption of the CL model and a "reasonable approximation of more complex relationships" (Ben-Akiva and Lerman 1985). A test was developed by Hausman and McFadden (1984) for evaluating the validity of the IIA assumption. Estimating the choice model using the CL specifications that exhibit the IIA assumption will lead to biased estimates if the IIA property is violated, therefore necessitating other model specifications.

One other way to test the IIA property is to estimate the heteroscedastic extreme value (HEV) by allowing all alternatives to have different scale parameters and to check whether these scale parameters are the same statistically. If the estimated scale parameters are the same statistically, then the CL model is fine._HEV modeling is based on the same random utility structure discussed above but simply relaxes the equal scale assumption across alternatives. In mathematical terms, the choice probability of alternative $i$ from a choice set $J$ by an individual $q$ is expressed as follows in HEV modeling:

$$
P_{i q}=\exp \left(\mu_{i} \beta^{\prime} X_{i q}\right) / \sum_{j=1}^{J} \exp \left(\mu_{j} \beta^{\prime} X_{j q}\right)
$$

where $\mu_{j}$ allows all alternatives with different scale parameters.

\subsection{CE design}

In CE design, 3 alternatives were provided: continued hospitalization, discharge home, or discharge to a postnatal care facility. A number of attributes and assigned levels, as indicated below, were designed to generate hypothetical scenarios. These attributes and their levels were determined after a broad literature review and careful discussion with specialists. Each alternative has 10 common attributes.

\section{$>$ Continued hospitalization}

- Distance from home to the facility: 30,60 , or $90 \mathrm{~km}$

- Length of stay in the facility: 5 days (fixed)

- Type of room in the facility: 1-, 2-, or 4-person room

- Number of examinations of the mother and baby by a physician: 1 (fixed)

- Time spent providing advice about feeding the baby: 5 minutes (fixed) 
- Number of childcare counseling sessions with a clinical psychologist: 0 (fixed)

- Childcare circle in the facility: Absent (fixed)

- Number of free 60-minute body massages provided to the mother: 0 (fixed)

- Family members can stay overnight during hospitalization: No (fixed)

- Total cost including delivery fees and other expenses: $¥ 450,000$, $¥ 500,000$, or $¥ 550,000$

$>$ Discharge home

- All levels are the same as presented for continued hospitalization, except length of stay is reduced to 2 days (fixed) and the total cost is $¥ 90,000$ less.

$>$ Discharge to a postnatal care facility

- Maternity facility: all levels are the same as presented for continued hospitalization, except length of stay is reduced to 2 days (fixed) and the total cost is the same as the postnatal care facility (listed below).

- Postnatal care facility:

o Distance from home to the facility: 3, 6, or $9 \mathrm{~km}$

o Length of stay in the facility: 3 , 4 , or 5 days

o Type of room in the facility: 1- or 2-person room

o Number of examinations of the mother and baby by a physician: 0 or 1

o Time spent providing advice about feeding the baby: 20, 30, or 40 minutes

o Number of childcare counseling sessions provided by a clinical psychologist: 1 or 2

o Childcare circle in the facility: Absent or present

o Number of free 60 -minute body massages provided to the mother: 1,2 , or 3

o Family members can stay the night during hospitalization: Yes or no

o Total cost for maternity and postnatal care facilities: $¥ 480,000$, $¥ 530,000$, or $¥ 580,000$

CE design is concerned with efficiently creating choice sets, i.e., combining the attribute levels into alternative profiles and combining alternative profiles into choice sets. If we applied a full factorial design, we would generate too many choice sets, which would be too cumbersome for respondents to answer. In this study, we used the D-optimal design approach for CE, which is based on multinomial logit modeling. The objective of D-optimal design is to extract the maximum amount of information from the respondents about the number of attributes, attribute levels, and other 
characteristics of the survey such as cost and length (Carlsson and Martinsson, 2003). We created 24 choice sets by running D-optimal design through Design-Expert 9.0 (Stat-Ease, Inc.). These choice sets were randomly divided into 3 versions, and each version of the questionnaire consisted of 8 choice sets. The respondents were asked to select their favorite location for postnatal care and answer several other questions related to their socioeconomic characteristics. An example of a choice set is presented in Table 1.

Table 1. Example of a choice set

\begin{tabular}{|c|c|c|c|c|}
\hline & \multirow[t]{2}{*}{$\begin{array}{c}\text { Continued } \\
\text { hospitalization }\end{array}$} & \multirow[t]{2}{*}{$\begin{array}{l}\text { Discharge } \\
\text { home }\end{array}$} & \multicolumn{2}{|c|}{$\begin{array}{l}\text { Discharge to postnatal care } \\
\text { facility }\end{array}$} \\
\hline & & & $\begin{array}{l}\text { Maternity } \\
\text { facility }\end{array}$ & $\begin{array}{c}\text { Postnatal care } \\
\text { facility }\end{array}$ \\
\hline Distance from home to facility & $60 \mathrm{~km}$ & $60 \mathrm{~km}$ & $60 \mathrm{~km}$ & $3 \mathrm{~km}$ \\
\hline Length of stay & 5 days & 2 days & 2 days & 5 days \\
\hline Type of room & 4-person room & 4-person room & 4-person room & 2-person room \\
\hline $\begin{array}{l}\text { Number of examinations by a } \\
\text { physician }\end{array}$ & 1 & 1 & 1 & 1 \\
\hline $\begin{array}{l}\text { Time spent providing } \\
\text { guidance about feeding the } \\
\text { baby }\end{array}$ & 5 minutes & 5 minutes & 5 minutes & 20 minutes \\
\hline $\begin{array}{l}\text { Number of childcare } \\
\text { counseling sessions by a } \\
\text { clinical psychologist }\end{array}$ & 0 & 0 & 0 & 2 \\
\hline $\begin{array}{l}\text { Presence or absence of a } \\
\text { childcare circle }\end{array}$ & Absence & Absence & Absence & Presence \\
\hline $\begin{array}{l}\text { Number of free } 60 \text {-minute } \\
\text { body massages }\end{array}$ & 0 & 0 & 0 & 1 \\
\hline $\begin{array}{l}\text { Family members can stay } \\
\text { overnight }\end{array}$ & No & No & No & No \\
\hline Total cost & $¥ 500,000$ & $¥ 410,000$ & $¥ 53$ & 000 \\
\hline $\begin{array}{l}\text { Please check the most } \\
\text { desirable alternative }\end{array}$ & $\square$ & $\square$ & & \\
\hline
\end{tabular}




\subsection{Sample and data collection}

The survey was conducted from July 2015 to January 2016 in Gero City, Gifu Prefecture, Japan with the purpose of examining women's preferences for a new style of postnatal care facility and its attributes. Questionnaires with self-addressed postage-paid envelopes were delivered to the mothers of toddlers at 9 nursery schools when they picked up their children. A total of 250 responses were mailed back. Among these responses, 5 responses did not answer any CE questions, leaving 1,960 valid observations for estimating CE data (245 responses $\times 8$ choice sets per response).

Table 2. Socioeconomic characteristics of the respondents $(n=250)$

\begin{tabular}{llllll}
\hline Characteristic & $\mathbf{n}$ & $\mathbf{\%}$ & Characteristic & $\mathbf{n}$ & $\mathbf{\%}$ \\
\hline Age, years & 1 & 0.4 & 2 & & \\
$20-24$ & 36 & 14.4 & 3 & 4 & 1.6 \\
$25-29$ & 83 & 33.2 & 4 & 22 & 8.8 \\
$30-34$ & 77 & 30.8 & 5 & 66 & 26.4 \\
$35-39$ & 53 & 21.2 & $\geq 6$ & 48 & 19.2 \\
$\geq 40$ & & & No. of children & 110 & 44.0 \\
Occupation & 89 & 35.6 & 1 & & \\
Full-time employed & 93 & 37.2 & 2 & 39 & 15.6 \\
Part-time employed & 61 & 24.4 & 3 & 122 & 48.8 \\
Full-time housewife & 7 & 2.8 & $\geq 4$ & 74 & 29.6 \\
Student or other occupation & & & Annual household income, $\mathbf{\# 1 , 0 0 0 , 0 0 0}$ & 15 & 6.0 \\
Education level & 7 & 2.8 & $<2$ & 7 & 2.8 \\
Junior high school & 80 & 32.0 & $2-3.99$ & 68 & 27.2 \\
Senior high school & 74 & 29.6 & $4-5.99$ & 86 & 34.4 \\
Technical degree & 87 & 34.8 & $6-7.99$ & 47 & 18.8 \\
Undergraduate degree & 1 & 0.4 & $8-9.99$ & 17 & 6.8 \\
Master’s degree & 1 & 0.4 & $\geq 10$ & 17 & 6.8 \\
Others & & & & & \\
\hline
\end{tabular}

A summary of the respondents' socioeconomic characteristics is provided in Table 2. Of 250 respondents, approximate $85 \%$ were $\geq 30$ years old. Regarding occupational status, $35.6 \%$ of the respondents were employed full-time, $37.2 \%$ were employed part-time, and $24.4 \%$ were full-time housewives. Eight-seven of 250 respondents (34.8\%) have a bachelor's degree and 1 respondent has a master's degree. In addition, $84.4 \%$ of the respondents have $\geq 2$ children, and about $90 \%$ of 
households have $\geq 4$ persons. Finally, the annual household income distribution of the respondents was similar to the 2013 official data of Gero City that were published by the Japanese Ministry of Internal Affairs and Communications (Japanese Ministry of Internal Affairs and Communications, 2013).

\section{Results and Discussion}

Table 3 presents the results estimated by the CL model. We used the Hausman test to evaluate IIA assumptions in this model. These results could not statistically reject the suitability of the IIA assumption. This was also confirmed by the estimated alternative scale parameters for continued hospitalization, discharge home, and discharge to a postnatal care facility that were statistically the same as HEV specification. Hence, in the current paper, we report the results based on the CL specifications.

As shown in Table 3, the 2 alternative-specific constants (continued hospitalization and discharge to a postnatal care facility) were significantly estimated with positive signs, suggesting that these are preferred by the respondents over the other alternative (discharge home). With respect to which of these 2 alternatives is more preferable, post-estimation testing indicated that discharge to a postnatal care facility was significantly larger than continued hospitalization $\left(\chi^{2}(1)=5.23 ; p=\right.$ 0.022), which implies that discharge to a postnatal care facility is more preferable.

In terms of the respondents' preferences towards the attributes of discharge to a postnatal care facility, all attributes were statistically significant except CareCenter_examination and CareCenter_stay. Among the significant attributes, length of stay, type of room, and total cost were estimated with negative signs, implying that respondents are favorable to a shorter stay in the postnatal care facility, staying with fewer people in the same room, and paying less to utilize postnatal care services. CareCenter_guidance, CareCenter_counseling, CareCenter_circle, and CareCenter_massage were estimated with positive signs, suggesting that respondents prefer longer sessions about feeding the baby, having more childcare counseling sessions with a clinical psychologist, having a childcare circle in the postnatal care facility, and having more free 60-minute body massages. In addition, while distance from home to the postnatal care facility (CareCenter_distance) was significantly estimated, its positive sign was not consistent with our prediction. In general, it is expected that mothers would prefer to utilize postnatal care services at a facility near their home, which is in line with the preference elicited by the significantly negative 
sign for Hospital_distance: i.e., mothers prefer to deliver their babies at the hospital near their home. However, there might be another possible explanation. Mothers could consider moving from the delivery hospital to the postnatal care facility as a different from simply going to the postnatal care facility. After 2 days in the hospital, the mother is released from the hospital if she does not select continued hospitalization, whether she goes directly home or to the postnatal care facility. Therefore, a mother may have 2 choices for how to utilize postnatal care services: 1) go to the postnatal care facility directly from the hospital, or 2) go home and utilize the day services of the facility. In this sense, a mother may not move to the postnatal care facility that it is near her home, but instead use a facility that is far from her home. This might be the reason why the sign of CareCenter_distance was estimated as negative.

Table 3. Estimation results of the CL model

\begin{tabular}{lcc}
\hline & Coefficient & Robust standard error \\
\hline Alternative-specific constants & & \\
Continued hospitalization & $5.895^{* *}$ & 0.497 \\
Discharge to a postnatal care facility & $7.021^{* *}$ & 0.877 \\
Alternative-specific attributes & & \\
Hospital_distance & $-0.011^{* *}$ & 0.002 \\
Hospital_bed & $-0.143^{* *}$ & 0.033 \\
Hospital_cost & $-0.070^{* *}$ & 0.009 \\
CareCenter_distance & $0.048^{* *}$ & 0.016 \\
CareCenter_days & $-0.167^{*}$ & 0.086 \\
CareCenter_bed & $-0.592^{* *}$ & 0.105 \\
CareCenter_examination & 0.068 & 0.079 \\
CareCenter_guidance & $0.007^{* *}$ & 0.002 \\
CareCenter_counseling & $0.161^{*}$ & 0.072 \\
CareCenter_circle & $0.215^{* *}$ & 0.080 \\
CareCenter_massage & $0.138^{* *}$ & 0.051 \\
CareCenter_stay & 0.123 & 0.085 \\
CareCenter_cost & $-0.083^{* *}$ & 0.013 \\
Log-likelihood convergence & -2741.842 & \\
Pseudo R & 0.172 & \\
Predictive power & $76.3 \%$ & \\
Observations & 1960 & \\
\hline
\end{tabular}

*This parameter is significantly different from 0 at a $5 \%$ level.

${ }^{* *}$ This parameter is significantly different from 0 at a $1 \%$ level. 
Table 4. Status quo scenario

\begin{tabular}{|c|c|c|c|c|}
\hline & \multirow[t]{2}{*}{$\begin{array}{c}\text { Continued } \\
\text { hospitalization }\end{array}$} & \multirow[t]{2}{*}{$\begin{array}{l}\text { Discharge } \\
\text { home }\end{array}$} & \multicolumn{2}{|c|}{$\begin{array}{l}\text { Discharge to a postnatal } \\
\text { care facility }\end{array}$} \\
\hline & & & $\begin{array}{l}\text { Maternity } \\
\text { facility }\end{array}$ & $\begin{array}{l}\text { Postnatal care } \\
\text { facility }\end{array}$ \\
\hline Distance from home to the facility & $60 \mathrm{~km}$ & $60 \mathrm{~km}$ & $60 \mathrm{~km}$ & $6 \mathrm{~km}$ \\
\hline Length of stay & 5 days & 2 days & 2 days & 3 days \\
\hline Type of room & 4-person room & 4-person room & 4-person room & 1-person room \\
\hline $\begin{array}{l}\text { Number of examinations by a } \\
\text { physician }\end{array}$ & 1 & 1 & 1 & 1 \\
\hline $\begin{array}{l}\text { Time spent providing guidance } \\
\text { about feeding the baby }\end{array}$ & 5 minutes & 5 minutes & 5 minutes & 10 minutes \\
\hline $\begin{array}{l}\text { Number of childcare counseling } \\
\text { sessions by a clinical psychologist }\end{array}$ & 0 & 0 & 0 & 1 \\
\hline Childcare circle in the facility & Absence & Absence & Absence & Presence \\
\hline $\begin{array}{l}\text { Number of free } 60 \text {-minute body } \\
\text { massages }\end{array}$ & 0 & 0 & 0 & 1 \\
\hline $\begin{array}{l}\text { Family members can stay } \\
\text { overnight }\end{array}$ & No & No & No & No \\
\hline Total cost & $¥ 430,000$ & $¥ 340,000$ & $¥ 50$ & 000 \\
\hline
\end{tabular}

Based on the estimated CL model and the status quo scenario presented in Table 4, the probability of selecting discharge to a postnatal care facility can be calculated using Equation (4). It should be noted that in the status quo scenario, the delivery hospital is Takayama Red Cross Hospital and the postnatal care facility is Gero Hospital. Therefore, the attribute levels for continued hospitalization and discharge to a postnatal care facility are the actual conditions (or plans for implementation) at Takayama Red Cross Hospital and Gero Hospital, respectively. As a result, the probability of choosing discharge to a postnatal care facility was calculated as 0.6432 . In addition, we considered 3 other scenarios by adjusting some attribute levels for discharge to a postnatal care facility. Scenario A lowers the total cost of discharge to a postnatal care facility from $¥ 500,000$ to $¥ 430,000$, but keeps all other values the same as the status quo scenario. Scenario B (1) extends the stay in the postnatal care facility from 3 days to 5 days, (2) extends the time spent providing guidance about feeding the baby from 10 minutes to 30 minutes, (3) increases the number of 
childcare counseling sessions from 1 to 2, (4) increases the number of free 60-minute body massages from 1 to 2, and (5) allows family members to stay overnight in the postnatal care facility. Scenario C combines the parameters of Scenarios A and B. Table 5 presents the choice probabilities of discharge to a postnatal care facility under these 3 scenarios. A value of $>0.70$ indicates high acceptance of the new postnatal care facility. Furthermore, choice probability under Scenario B is higher than under Scenario A, implying that upgrading service is more useful than lowering cost in order to attract mothers to the facility.

Table 5. Choice probabilities of discharge to a postnatal care facility under different scenarios

\begin{tabular}{|c|c|c|c|}
\hline Status quo & Scenario A & Scenario B & Scenario C \\
\hline 0.6432 & 0.7201 & 0.7391 & 0.8018 \\
\hline
\end{tabular}

Note: Scenario A lowers the total cost from $¥ 500,000$ to $¥ 430,000$. Scenario B upgrades the service level by (1) extending the stay in the postnatal care facility from 3 to 5 days, (2) extends the time spent providing advice about feeding the baby from 10 minutes to 30 minutes, (3) increases the number of childcare counseling sessions from 1 to 2, (4) increases the number of free 60-minute body massages from 1 to 2, and (5) allows family members to stay overnight in the postnatal care facility. Scenario C combines the attributes of Scenarios A and B.

\section{Conclusion}

In this paper, a new style of postnatal care facility that combines medical and nonmedical support is considered. Contrary to most existing postnatal care facilities in Japan, this new postnatal care facility accepts the puerperant from the cooperating maternity facility within 2 days after giving birth. We conducted a hypothetical-choice experiment to investigate whether this new postnatal care facility would be accepted by pregnant women in Gero City and how they would evaluate different kinds of postnatal care services. We found that after 2 days in the hospital, the respondents preferred to move to the new postnatal care facility over the other alternatives (continued hospitalization or discharge home). In addition, the estimated choice probability of selecting discharge to a postnatal care facility changed from 0.6432 under the status quo scenario to 0.8018 under Scenario C, which indicates a high level of acceptance of the new postnatal care facility in Gero City. However, a more systematic cost-benefit analysis that takes into account the benefits and costs of the consumers and suppliers needs to be conducted.

The local government and/or residents might oppose a postnatal care facility because, by 
changing the role of Gero Hospital, giving birth in Gero City will be impossible afterward. This is, in part, similar to the so-called status quo bias. Status quo bias is an emotional bias that takes the current baseline (or status quo) as a reference point, and any change from that baseline is perceived as a loss (for more details, see in Samuelson and Zeckhauser (1988)). Therefore, convincing the local government and/or residents to overcome this bias will be the most important challenge in practice.

\section{Acknowledgements}

This research is financially supported by the Japan Society for the Promotion of Science (JSPS KAKENHI Grant Nos. 24243028, 16K13354, and 17H00980) and the Research Institute for Humanity and Nature (RIHN Project No. 40410000). All views expressed in this article and any errors are the sole responsibility of the authors.

\section{References}

Bastos MH, McCourt C: Morbidity during the postnatal period: impact on women and society. In Byrom S, Edwards G, Bick D, editors. Essential Midwifery Practice: Postnatal Care. Oxford, Wiley-Blackwell 2010, p.113-137.

Beake S, Rose V, Bick D, Weavers A, Wray J: A qualitative study of the experiences and expectations of women receiving in-patient postnatal care in one English maternity unit. $B M C$ Pregnancy and Childbirth 2010, 10:70.

Bowers J, Cheyne H: Reducing the length of postnatal hospital stay: implications for cost and quality of care. BMC Health Services Research 2016, 16:16.

Carlsson F, Martinsson P: Design techniques for stated preference methods in health economics. Health Economics 2003, 12:281-294.

Fukai S, Aoki N, Ootake M, Oomagari M: Survey on current situations and needs for child care support in Arakawa. Journal of the National Institute of Public Health 2015, 64(2):112-115. (in Japanese)

Ichikawa Y, Iseki A: Cognition of puerpera about early discharge. Mie Nursing Journal 2013, 15(1):61-67. (in Japanese) 
Japanese Ministry of Internal Affairs and Communications: 2013 Housing and Land Survey. (in Japanese)

Johansson K, Aarts C, Darj E: First-time parents' experiences of home-based postnatal care in Sweden. Upsala Journal of Medical Sciences 2010, 115:131-137.

Kawahara N, Kamiya S, Emisu F: Needs related to early hospital discharge after giving birth. Bulletin of Aichi Prefectural College of Nursing \& Health 2012, 18:39-46

Kurth E, Spichiger E, Stutz EZ, Biedermann J, Hösli I, Kennedy HP: Crying babies, tired mothers - challenges of the postnatal hospital stay: an interpretive phenomenological study. $B M C$ Pregnancy and Childbirth 2010, 10:21.

Louviere JJ, Hensher DA, Swait JD: Stated Choice Methods: Analysis and Applications. Cambridge: Cambridge University Press; 2000.

McFadden D: Conditional Logit Analysis of Qualitative Variables in Econometrics. In Zarembka P, editor. Frontiers in Econometrics. New York, Academic Press 1974, p.105-142.

Ononokpono DN, Odimegwu CO, Imasiku ENS, Adedini SA: Does it really matter where women live? A multilevel analysis of the determinants of postnatal care in Nigeria. Maternal and Child Health Journal 2014, 18:950-959.

Rayner J, NcLachlan HL, Forster DA, Peters L, Yelland J: A statewide review of postnatal care in private hospitals in Victoria, Australia. BMC Pregnancy and Childbirth 2010, 10:26.

Sakanashi K, Katsukawa Y, Usui M, Nabeta M, Ohga A, Nagai S: Preference regarding conditions for postpartum early discharge and support system: View of medical profession. Yokohama Journal of Nursing 2011, 4(1):71-77. (in Japanese)

Samuelson W, Zeckhauser R: Status quo bias in decision making. Journal of Risk and Uncertainty 1988, 1(1):7-59.

Shen J: A review of stated choice method. International Public Policy Studies 2006, 10(2):97-121. 
Slade P, Morrell CJ, Rigby A, Ricci K, Spittlehouse J, Brugha TS: Postnatal women's experiences of management of depressive symptoms: a qualitative study. British Journal of General Practice 2010, 60:e440-e448.

Smith LFP: Postnatal care: development of a psychometric multidimensional satisfaction questionnaire (the WOMBPNSQ) to assess women's views. British Journal of General Practice 2010, 61:e628-e637.

Tao F, Huang K, Long X, Tolhurst R, Raven J: Low postnatal care rates in two rural counties in Anhui Province, China: Perceptions of key stakeholders. Midwifery 2011, 27:707-715. 